\title{
Primer Design for PCR and Sequencing in High-Throughput Analysis of SNPs
}

\author{
Ellen F. Vieux ${ }^{1}$, Pui-Yan Kwok ${ }^{1,2}$, and Raymond D. Miller ${ }^{1}$ \\ ${ }^{1}$ Division of Dermatology, Washington University School of Medicine, St. Louis, MO; \\ ${ }^{2}$ Current affiliation: Cardiovascular Research Institute, University of California, San Francisco, CA, USA
}

BioTechniques 32: S28-S32 (June 2002)

\begin{abstract}
To achieve high-throughput analysis of allele frequencies in human $S N P s$, we have developed automated methods for designing PCR and DNA sequencing primers. We found we could run the PCR assays at quite stringent, uniform conditions. The design process used freely available databases, including dbSNP, SNPper, and TSC, and publicly available software including RepeatMasker and Primer3. We describe parameters for the software and other considerations that increase experimental success. As anticipated, some assays failed at the design stage due primarily to the genomic locations of repetitive sequences, extreme GC content regions, or lack of sufficient sequence. However, over 23000 assays, including 96\% of those recently analyzed, have been experimentally successful. Similar design methods could be used for PCR assays in any organism with substantial available sequence.
\end{abstract}

\section{INTRODUCTION}

The detection of large numbers of candidate single nucleotide polymorphisms (SNPs) has been an important aspect of human genome investigation. The majority of these SNPs were found by comparing sequences of overlapping genomic clones or by comparison of new sequences to the reference draft sequence $(4,10)$. For brevity, we refer to candidate SNPs simply as SNPs, but it should be noted that for any one candidate, further investigation is required to determine whether or not it is polymorphic in one or more samples. While approximately $66 \%$ of SNPs (mainly autosomal) are common (rare allele $\geq 0.2$ ) in at least one sample, about $12 \%$ have low levels of variation and $22 \%$ have no detectable variation (Reference 4 and unpublished results). While most SNPs are expected to have little or no phenotypic effects, often a particular SNP will be in linkage disequilibrium with many other SNPs (e.g., Reference 9). Some SNPs undoubtedly will be important for health, including susceptibility to various diseases and metabolism of drugs.

To aid in the development of SNPs as research tools, our group has been determining the allele frequencies of a large number of SNPs in three different population samples. To analyze the SNPs, we have developed and performed PCR on each SNP using DNA from a reference individual and the samples of pooled DNA (42 individuals, each from African Americans, Asian Americans, or European Americans), sequenced the PCR products, and estimated the allele frequencies in the samples by determining the contributions of each allele to the electropherogram (3). It was essential that (i) the assays were conducted us- ing consistent conditions to enable high-throughput analysis, (ii) the assays were conducted under very stringent conditions to minimize spurious products which would obscure DNA sequencing, and (iii) most of the designed assays were experimentally successful. Using publicly available databases and software, we assembled a design process that met these criteria. In our process, one of the PCR primers was also used for cycle sequencing of the PCR product, using fluorescent dye terminators, so only two primers were required; these were relatively inexpensive since they were unlabeled. With slight modifications, the method could be applied to many organisms. In this paper we describe our assay design and give examples of the results.

\section{MATERIALS AND METHODS}

The SNPs analyzed in this project were obtained through a variety of means including choosing sets based on spacing along a genome assembly and receiving sets submitted by investigators interested in particular genomic regions. Each analyzed SNP was initially communicated to the project as a unique identifier, an rs number (non-redundant identifier from public database dbSNP: http://www.ncbi.nlm.nih.gov/SNP/), (8), an ss number (accession number from dbSNP), or a TSC identifier from The SNP Consortium (TSC: http://snp.cshl.org/). The SNP then progressed through a series of steps or pipeline encompassing assay design, experimental analysis, and reporting of results (Table 1).

\section{Obtaining the Sequence}

We obtained the sequence surrounding a SNP site from one of three sources. The first source was dbSNP. We submitted lists of rs or ss numbers to this database in batch format through their Web interface, and the surrounding sequence of the longest acquisition was returned. SNP mapping information, updated with each genome assembly build, was downloaded from the $\mathrm{ftp}$ site. A second source used was SNPper, http:// bio.chip.org:8080/bio/ (5), where submission of a batch of rs numbers allowed return of up to $1000 \mathrm{bp}$ on either side of the SNP. SNPper determined flanking sequence from a genome assembly, allowing only uniquely mapped candidates to be returned. A third source used was The SNP Consortium, whose personnel kindly provided surrounding sequence for TSC accessions, which have been uniquely mapped. Each of the three resources returned sequence in FASTA format with some variation 
Table 1. SNP Characterization Pipeline

\begin{tabular}{|ll|}
\hline Step & Operation \\
\hline 1 & Determine SNP Candidates \\
2 & Design Primers \\
a & Obtain sequence \\
b & Mark repetitive sequence \\
c & Choose primers using set parameters \\
d & Final processing \\
3 & Acquire data \\
a & Obtain primers \\
b & PCR \\
c & Sequence \\
d & Capillary electrophoresis \\
4 & Analyze data and troubleshoot if necessary \\
5 & Report results \\
\hline
\end{tabular}

in syntax. For subsequent steps the returned sequences were converted to a consistent format for identification of the SNP within the sequence and a consistent header format designating the rs number and SNP position.

\section{Marking Repetitive Sequences}

For specificity of PCR products we attempted to avoid picking primers within repeated regions, including identified high or low copy number repeats. The returned sequences containing the SNP sites were saved for later steps, and then analyzed with the software RepeatMasker, http://ftp.genome.washington.edu/ $\mathrm{RM} /$. RepeatMasker produced a "masked" version of the sequence, with the bases for identified repeats converted to " $N$ " in FASTA format. The masked sequences and input parameters were then converted to BoulderIO format for submission to the program for choosing primers.

\section{Choosing Primers Using Set Parameters}

To choose the primers, we used the software Primer3, release 0.9, Unix version (Steve Rozen, Helen J. Skaletsky, copyright 1996, 1997, 1998; code available at http://www-genome.wi. mit.edu/genome_software/). An online version is also available through the World Wide Web (6). The input parameters we used for Primer3 are shown (Table 2). Since many SNPs were available for analysis, our strategy was to use stringent design criteria to maximize experimental success, expecting a higher design failure rate than may be necessary for some experiments.

In using Primer3 for PCR design, Beasley et al. found that there was a higher experimental failure rate with primers $20 \mathrm{bp}$ or less (or related, if the primer GC content was greater than $50 \%$ ), and they accordingly adjusted primer size and GC content parameters (1). They used an optimal primer $\mathrm{T}_{\mathrm{m}}$ of $62^{\circ} \mathrm{C}$, whereas we previously had good success with an optimal $\mathrm{T}_{\mathrm{m}}$ of $55^{\circ} \mathrm{C}$, and we continued to use the latter value. The first parameter, "TARGET", was set with a length of $50 \mathrm{bp}$, beginning 25 bp upstream of the SNP site, thus effectively excluding this region as a source of primers and preventing overlap of the primers with the SNP. Parameters 2-4 set the primer size and were taken from
Table 2. Parameter Values for Primer3

\begin{tabular}{|clr|}
\hline & Parameter & Value \\
\hline 1 & TARGET & $x, y$ \\
2 & PRIMER_OPT_SIZE & 23 \\
3 & PRIMER_MAX_SIZE & 26 \\
4 & PRIMER_MIN_SIZE & 20 \\
5 & PRIMER_OPT_TM & 55 \\
6 & PRIMER_MAX_TM & 56 \\
7 & PRIMER_MIN_TM & 54 \\
8 & PRIMER_PRODUCT_SIZE_RANGE & $150-400$ \\
9 & PRIMER_PRODUCT_OPT_SIZE & 300 \\
10 & PRIMER_PAIR_WT_PRODUCT_SIZE_LT & 0.20 \\
11 & PRIMER_PAIR_WT_PRODUCT_SIZE_GT & 0.20 \\
12 & PRIMER_MIN_GC & 20 \\
13 & PRIMER_MAX_GC & 50 \\
14 & PRIMER_SALT_CONC & 50 \\
15 & PRIMER_SELF_ANY & 8 \\
16 & PRIMER_SELF_END & 3 \\
17 & PRIMER_GC_CLAMP & 0 \\
18 & PRIMER_MAX_END_STABILITY & 8 \\
19 & PRIMER_DNA_CONC & 40 \\
20 & PRIMER_NUM_RETURN & 1 \\
& x, starting position of target (bp); y, length of target $=50$ bp \\
Units: size (bp), T ( ${ }^{\circ}$ C), weight (fraction), GC (\%), concen- \\
tration (mM), return (primer pairs). \\
\hline
\end{tabular}

Beasley et al., except we found that decreasing the minimal size to $20 \mathrm{bp}$ with our reduced $\mathrm{T}_{\mathrm{m}}$ provided an increased rate of success for primer design with no apparent increase of experimental failure. Parameters $5-7$ set the $T_{m}$ to $55 \pm 1^{\circ} \mathrm{C}$. Parameters $8-11$ set the product size range to $150-400 \mathrm{bp}$. The products were long enough to usually permit an optimal spacing interval of $\geq 100$ bp between the first base of the sequencing primer and the SNP. Compared with longer PCR products, this range and flexibility enabled many assays to be successful, while still permitting a decreased sequencing time and minimizing the occurrence of unknown insertion-deletion events between the sequencing primer and the SNP. Parameters 12-18 were from Beasley et al. For parameter 19, the effective primer concentration, we used a value near the default value. Parameter 20 caused only one pair of primers to be reported, thus simplifying later steps.

\section{Final Processing}

After a primer pair was chosen, we did additional analysis to choose the sequencing primer, screening out some situations prone to failures. Long runs of T's, A's, GT's, and CA's decreased the fluorescent intensity of longer products and were likely to be polymorphic for insertion/deletion events. If the primer used for sequencing was less than $100 \mathrm{bp}$ from the candidate site, spurious peaks in the electropherograms could obscure the peaks used for allele frequency estimation. Accordingly, we used a simple algorithm, executed using a Perl script, to check conditions in sequences that were not repeat-masked. The left primer was exam- 
ined to see whether it passed or failed (beginning of the primer sequence less than $100 \mathrm{bp}$ from the candidate or stretches of 8 or more T's or A's, or 10 or more GTs or CA's). The right primer was similarly examined. If both primers failed, the assay was designated as failed. If one primer passed and one failed, the former was designated the sequencing primer. If both passed, the one closest to the candidate site was designated the sequencing primer.

\section{PCR and Sequencing Conditions}

To make the assays stringent, we avoided choosing primers within repetitive sequence; we used a hot start DNA polymerase; we used short extension times; and we performed the PCR annealing steps $3^{\circ} \mathrm{C}$ above the design $\mathrm{T}_{\mathrm{m}}$ of $55 \pm 1^{\circ} \mathrm{C}$. For a particular PCR cycle, the actual $\mathrm{T}_{\mathrm{m}}$ for primer and template was expected to be a function of the concentration of both components, with the concentration changing throughout the reactions. PCR amplification using higher annealing temperatures than the nominal $\mathrm{T}_{\mathrm{m}}$ have been reported (e.g., 7).

Human genomic DNA (4 ng) from Coriell Institute, Camden, NJ, USA, was amplified in $10-\mu \mathrm{L}$ reaction mixtures, each containing 0.15 U JumpStart ${ }^{\mathrm{TM}}$ Taq DNA polymerase (SigmaAldrich, St. Louis, MO, USA), $50 \mathrm{mM} \mathrm{KCl,} 10 \mathrm{mM}$ Tris- $\mathrm{HCl}$, $\mathrm{pH}$ 8.3, $3.5 \mathrm{mM} \mathrm{MgCl} 2,100 \mathrm{nM}$ each of the four dNTPs, 1.0 $\mu \mathrm{M}$ primer- 1 and $0.1 \mu \mathrm{M}$ primer- 2 . We have found addition of extra primer-1 (used for sequencing) at this stage removed the need for later addition of a primer for cycle sequencing (manuscript in preparation). The thermocycling protocol comprised an initial step at $95^{\circ} \mathrm{C}$ for 2 minutes to activate the polymerase, then 35 cycles of denaturation at $92^{\circ} \mathrm{C}$ for 10 seconds, annealing at $58^{\circ} \mathrm{C}$ for 20 seconds, and extension at $68^{\circ} \mathrm{C}$ for 30 seconds, followed by a final extension at $68^{\circ} \mathrm{C}$ for 10 minutes.

Cycle DNA sequencing was conducted according to the protocol of the dye manufacturer (Applied Biosystems). Briefly, 2.5 $\mu \mathrm{L}$ of the PCR mixture was added to $6.5 \mu \mathrm{L}$ of water, $2.0 \mu \mathrm{L}$ of BigDye version $3 \mathrm{mix}$, and $1.0 \mu \mathrm{L}$ of $5 \times$ sequencing buffer. The thermoycling protocol comprised an initial step at $96^{\circ} \mathrm{C}$ for 2 minutes, then 25 cycles of denaturation at $96^{\circ} \mathrm{C}$ for 15 seconds, annealing at $50^{\circ} \mathrm{C}$ for 1 second, and extension at $68^{\circ} \mathrm{C}$ for 4 minutes. Extra dyes were removed from the sequencing reactions according to the manufacturer's protocols, using columns in 96or 384-well plate format (Princeton Separation, Philadelphia, PA or Genetix, St. James, NY, USA). The samples were electrophoresed on a 3700 DNA Sequencer (Applied Biosystems).

\section{RESULTS AND DISCUSSION}

Our SNP characterization pipeline has produced very satisfactory experimental results (e.g., Figure 1). Allele frequencies found using this pipeline have been deposited in the dbSNP and TSC databases. Bulk downloads of our characterization of over 23000 SNPs are available at ftp://snp.cshl.org/pub/SNP/ frequency and http://snp.wustl.edu.

To illustrate results from the assay design, two processed groups of SNPs are shown, one utilizing sequences from dbSNP (group A) and the other from SNPper returning $600 \mathrm{bp}$ flanking sequence (group B), which on average provided more flanking sequence than dbSNP (Table 3). As mentioned, our strategy was to have very consistent, successful assay conditions, even at the expense of failures at the design stage. Group A had a $43 \%$ success rate of assay design and group B a 69\% success rate, reflecting the advantage of having additional flanking sequence. Analysis with Primer3 tested many primers for each SNP, first the left primers, then the right. If one or more left and right primers were found, the program checked whether any produce the correctly sized products. The largest reason for failure to pick a left or right primer was due to extreme local genomic GC content, followed by long stretches of N's in the sequence (from masking repeats). For example, for all the sequences of group A, 913006 potential primers were examined. Some $41 \%$ of failures were due to GC content, $29 \%$ exceeded the maximum $\mathrm{T}_{\mathrm{m}}$, $15 \%$ did not meet the minimum $\mathrm{T}_{\mathrm{m}}, 14 \%$ had poly-N's, and about 1\% failed for other causes. For group B, 796191 primers were examined. Some $38 \%$ of failures were due to GC content, $34 \%$ exceeded the maximum $\mathrm{T}_{\mathrm{m}}, 17 \%$ did not meet the minimum $\mathrm{T}_{\mathrm{m}}, 9 \%$ had poly-N's, and about $2 \%$ failed for other causes. While these distributions are similar, clearly there are differences in the samples in GC and repeat content. When the right and left primers were matched into pairs, similar percentages of SNPs failed in assay design for groups A or B because there was no possible product between $150-400 \mathrm{bp}, 13 \%$ and $15 \%$, respectively. Some additional failures occurred at the post-processing step, $6 \%$ and $3 \%$ for groups A and B, respectively; the major reason for failure was that neither primer was $\geq 100 \mathrm{bp}$ from the SNP. Although local genomic context affected assay design results, the additional flanking sequence available for group B clearly increased the percentage of SNPs for which assays could be designed, primarily because Primer 3 had a better chance of successfully picking a left and right PCR primer.

In spite of local variations in GC and repeat content in human DNA, the results suggest that the success rate of assay design for our criteria could be increased by obtaining 375 bp of

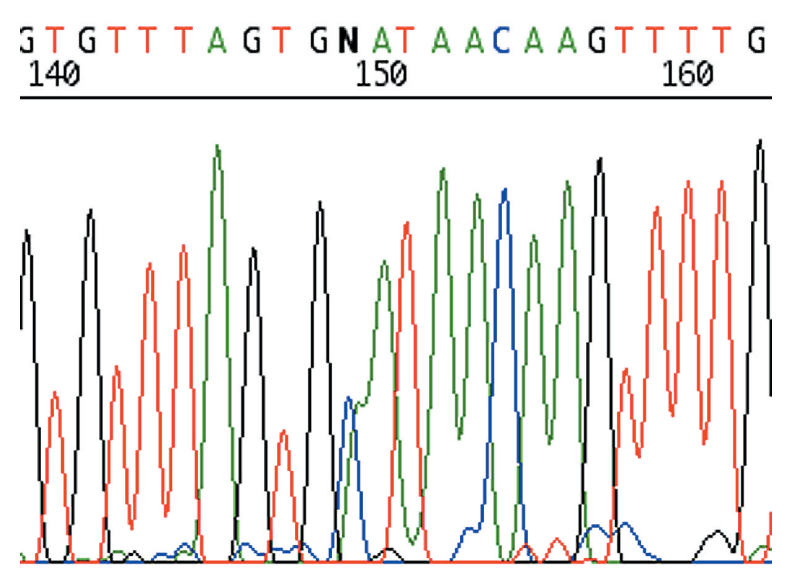

Figure 1. Electropherogram of human SNP rs1894466. The SNP is located on the $\mathrm{X}$ chromosome, and results, which use the assay design described, are from one polymorphism discovery panel DNA. Primers used were p1: 5'-CTTCTTATTACTGAAACTAATGCTGT-3' and p2: 5'-CGGCCAAAACTGACTAACTAT $-3^{\prime}$. The individual was a heterozygote for the predicted $[\mathrm{A} / \mathrm{C}]$ candidate site. Results of the assay using pooled DNA samples demonstrated the candidate is polymorphic (frequency of $\mathrm{C}$ in African Americans, Asians Americans, and European Americans is respectively 0, 0.60, and 0.30; data not shown). 
Table 3. Choosing Primers

\begin{tabular}{|lcc|}
\hline & \multicolumn{2}{c|}{ Group (\%) } \\
Result & A & B \\
\hline Success & 43.4 & 68.9 \\
Failures & & \\
$\quad$ Left and/or right primer & 37.3 & 13.1 \\
$\quad$ Product size & 13.3 & 15.0 \\
$\quad$ Post process & 6.1 & 2.9 \\
$\quad$ Total & 100 & 100 \\
\cline { 2 - 3 } Number attempted & 445 & 206 \\
\hline
\end{tabular}

Table 4. Assay Success

\begin{tabular}{|lr|}
\hline Result & (\%) \\
\hline Success & \\
Variation & 64 \\
$\quad$ No variation & 30 \\
Some data missing & 2 \\
$\quad$ Total & 96 \\
Failure & 3 \\
$\quad$ No PCR & 1 \\
Repeat sequence & 4 \\
$\quad$ Total & 672 \\
Number attempted & \\
Note: X chromosome SNPs & \\
\hline
\end{tabular}

flanking sequence on either side of the SNP. The 375 bp plus 25 bp from half of the excluded target permits a 400 bp maximum allowed PCR product, reducing failures compared with shorter sequences. A slight improvement in success rate for the postprocessing stage could be obtained by increasing the number of primer pairs returned by Primer3, thus increasing the chance for a successful assay. Not all failed assays could be helped by additional flanking sequence and more returned primer pairs, because in some locations the repeats or local GC content preclude picking primers, unless the design criteria are altered. However, many assays can be designed using available sequence and standard criteria. For humans, we have designed and made publicly available 770042 PCR assays at http://snp.wustl.edu.

To estimate the experimental success rate of this design process, we consider 672 assays, all for SNPs located on the X chromosome (Table 4). These were analyzed in a 384-well plate format using liquid handling equipment, which in our experience increased the experimental success rate. In spite of our very stringent PCR conditions, $96 \%$ of these assays were successful. Some 3\% were failures due to lack of a PCR product, and $1 \%$ failed due to apparent amplification of undocumented repeat regions. Incidentally, as we have previously observed, the percentage of SNPs with no observed variation $(30 \%)$ was increased in this X chromosome group as compared with groups of SNPs primarily from autosomes (e.g., 22\%; unpublished results). We have also successfully used similar PCR assay designs for genotyping by single base extension assays using fluorescent polarization (Reference 2 and unpublished results). For any organism having substantial available draft-or-better sequence, adapta- tions of this method of PCR design and sequencing should allow experimental access to population genomic information.

\section{ACKNOWLEDGMENTS}

We thank Elizabeth Gatrell Lovins for the figure and Patricia Taillon-Miller for comments on the manuscript. This work was funded in part by grants from the NIH (HG01720 and GM63340) and The SNP Consortium.

\section{REFERENCES}

1.Beasley, E.M., R.M. Myers, D.R. Cox, and L.C. Lazzeroni. 1999. Statistical refinement of primer design parameters, p. 55-71. In M.A. Innis, D.M. Gelfand, and J.J. Sninsky (Eds.), PCR Applications. Academic Press, San Diego.

2.Hsu, T.M., X. Chen, S. Duan, R.D. Miller, and P.Y. Kwok. 2001. Universal SNP genotyping assay with fluorescence polarization detection. BioTechniques 31:560-568.

3.Kwok, P.Y., C. Carlson, T.D. Yager, W. Ankener, and D.A. Nickerson. 1994. Comparative analysis of human DNA variations by fluorescence-based sequencing of PCR products. Genomics 23:138-144.

4.Marth, G., R. Yeh, M. Minton, R. Donaldson, Q. Li, S. Duan, R. Davenport, R.D. Miller, and P.Y. Kwok. 2001. Single-nucleotide polymorphisms in the public domain: how useful are they? Nat. Genet. 27:371-372.

5.Riva, A.A. and I.S. Kohane. 2001. A web-based tool to retrieve human genome polymorphisms from public databases. Proc. AMIA Symp. 2001:558-562.

6.Rozen, S. and H. Skaletsky. 2000. Primer3 on the WWW for general users and for biologist programmers. Methods Mol. Biol. 132:365-386.

7.Rychlik, W., W.J. Spencer, and R.E. Rhoads. 1990. Optimization of the annealing temperature for DNA amplification in vitro. Nucleic Acids Res. 18:6409-6412.

8.Smigielski, E.M., K. Sirotkin, M. Ward, and S.T. Sherry. 2000. dbSNP: a database of single nucleotide polymorphisms. Nucleic Acids Res. 28:352-355.

9.Taillon-Miller, P., I. Bauer-Sardina, N.L. Saccone, J. Putzel, T. Laitinen, A. Cao, J. Kere, G. Pilia, et al. 2000. Juxtaposed regions of extensive and minimal linkage disequilibrium in human $\mathrm{Xq}_{\mathrm{q}} 2$ and Xq28. Nat. Genet. 25:324-328.

10.The_International_SNP_Map_Working_Group. 2001. A map of human genome sequence variation containing 1.42 million single nucleotide polymorphisms. Nature 409:928-933.

Address correspondence to:

Dr. Raymond D. Miller

Washington University School of Medicine

Division of Dermatology, Box 8123

660 S. Euclid Ave.

St. Louis, MO 63110, USA

e-mail:rmiller@psts.wustl.edu 